\title{
Upper airway cooling reduces upper airway resistance in anaesthetized young guinea-pigs
}

\author{
A.K. Curran*, K.D. O'Halloran**, A. Bradford+
}

Upper airway cooling reduces upper airway resistance in anaesthetized young guinea-pigs. A.K. Curran, K. D. O'Halloran, A. Bradford. (OERS Journals Ltd 1998.

ABSTRACT: In adults, the upper airway (UA) contains a variety of receptors including cold receptors, which evoke reflex effects on ventilation and UA dilator muscle activity, which may be important in the regulation of UA patency. However, very little is known about UA receptors in young animals, and the effects of UA cooling on UA dilator muscle activity and resistance have not been studied.

A constant flow of warm or cool air was applied to the isolated UA in anaesthetized, vagotomized young guinea-pigs breathing spontaneously through a low-cervical tracheostomy while ventilation, UA resistance and geniohyoid muscle electromyographic activity were recorded.

Cooling caused an inhibition of breathing, a reduction in UA resistance and an excitation of geniohyoid muscle activity. Topical anaesthesia of the UA or sealing the nose and cutting the superior laryngeal and glossopharyngeal nerves abolished the ventilatory and geniohyoid muscle responses but not the fall in UA resistance.

It is concluded that upper airway cooling reflexly inhibits breathing and excites geniohyoid muscle activity. Cooling also reduces upper airway resistance by an effect which is not of reflex origin, possibly by reducing upper airway mucosal blood flow. Eur Respir J 1998; 11: 1257-1262.
*Dept of Preventive Medicine, and **Dept of Comparative Biosciences, School of Veterinary Medicine, University of Wisconsin at Madison, USA. +Dept of Physiology, Royal College of Surgeons in Ireland, Dublin, Ireland

Correspondence: A. Bradford

Dept of Physiology

Royal College of Surgeons in Ireland St Stephen's Green

Dublin 2

Ireland

Fax: 35314022447

Keywords: Airway cooling, airway resistance, geniohyoid

Received: July 91997

Accepted after revision February 271998

This work was supported by grants from the Health Research Board (Ireland), the Royal College of Surgeons in Ireland and the Wellcome Trust.
In adult animals, the upper airway (UA) has been shown to contain receptors sensitive to UA cooling [1-5]. The application of cool air to the isolated UA inhibits breathing [6-9] and this effect is abolished by laryngeal anaesthesia [6] or by cutting the superior laryngeal nerves [7-9]. UA cooling has also been shown to modify UA dilator muscle activity [7-11] and this effect was also abolished by superior laryngeal nerve section $[7,9,11]$. In adult rats, moderate UA cooling has been shown to increase UA dilator muscle activity and reduce UA resistance $[8,9]$, suggesting that changes in UA temperature may play a role in the control of UA patency.

The effects of UA cooling on UA muscle activity and UA resistance have not been investigated in young animals. Little is known about UA receptors in young animals, although superior laryngeal nerve afferents responding to UA pressure, airflow, UA movement and/or muscle activity have been described in neonatal dogs [12] and to water in neonatal dogs [13] and neonatal sheep, cats and monkeys [14]. UA cooling has been shown to inhibit breathing in neonatal dogs [15] and guinea-pigs [16] and possibly in neonatal cats [17], and these effects were abolished by laryngeal anaesthesia [17] or superior laryngeal nerve section $[15,16]$. Menthol, a specific cold receptor stimulant, also inhibits breathing in neonatal dogs [18]. It has been suggested that the ventilatory depression caused by cooling and laryngeal reflex responses to water is greater in neonates than in adults $[12,13,17]$.

The purpose of the present study was to examine the effects of UA cooling on UA dilator muscle activity and on UA resistance in young guinea-pigs. The young guineapig is known to be neurologically more mature than several other species [19]. However, it has been shown previously that UA reflex responses are very different in 10-14day-old compared with adult guinea-pigs [20]. There are also maturational differences in local airway responses, since exsanguination-induced bronchoconstriction has been shown to be greater in 15-day-old than in adult guineapigs [21].

\section{Materials and methods}

The procedure for isolation of the UA has been described previously [16]. In brief, 17 young guinea-pigs (Biological Laboratories Europe Ltd., Carrentrila, Ireland) aged 15.7 \pm 0.4 days with a body weight of $197.8 \pm 7.3 \mathrm{~g}$ (mean $\pm \mathrm{sD})$ were anaesthetised using urethane $\left(2 \mathrm{~g} \cdot \mathrm{kg}^{-1}\right.$ i.p. $)$ and placed supine on a thermostatically controlled heating pad to maintain body temperature at $37^{\circ} \mathrm{C}$. The fur was removed from the ventral aspect of the neck and a midline incision was made, exposing the trachea. With the aid of a binocular microscope (Leica, Wild MC3, Heerbrugg, Switzerland) a low cervical tracheostomy was performed through which the animal could breathe spontaneously. Tracheal airflow was continuously recorded using a heated pneumotachograph (Hans Rudolph, KS, USA) and differential pressure transducer (Validyne DP 15, North Ridge, CA, USA) placed in series with the tracheal cannula and the signal was integrated to give tidal volume. A common 
carotid artery was cannulated and connected to a suitably calibrated pressure transducer (Validyne DP 215) for the measurement of arterial blood pressure. A jugular vein was cannulated for supplemental doses of anaesthetic as required.

A high-cervical tracheostomy was performed through which a cannula was inserted cranially and extended to lie just below the level of the cricoid cartilage. A high pressure source produced a steady flow of $5-10 \mathrm{~mL} \cdot \mathrm{s}^{-1}$, of warmed, humidified air or cool, room-humidity air which was delivered to the UA in an expiratory direction and passed over the larynx and pharynx to exit either through the nose and mouth (nose open) or through the mouth only (nose sealed). UA airflow was recorded using a pneumotachograph (Hans Rudolph) placed before the warm and cool air circuit and connected to a pressure transducer (Validyne DP 15) calibrated at the same temperature as this airflow. The warm flow passed first through a humidification chamber which consisted of a flask containing a small am-ount of warm water. From there the flow passed through a coil of tubing which was contained within a water jacket. The temperature of the air was determined by the temperature of the water circulating through this jacket. The coil exited the jacket and was connected to the UA catheter through a three-way stopcock. The other arm of the stopcock was connected to the cool airflow system. This was identical to the warm flow system except that the humidification chamber had no water and the heating jacket was held at a lower temperature. The UA pressure and airflow temperature were measured between the stopcock and the UA catheter. The temperature of the UA airflow was monitored using a thermocouple microprobe (Physitemp, Model TH8, with probe IT21, time constant $0.08 \mathrm{~s}$, Clifton, USA) extended into the lumen of the UA cannula through a sidearm. Subglottic UA pressure was measured using a pressure transducer (Validyne DP 15) connected to a sidearm of the UA cannula. Mean values of UA pressure and flow were used to calculate UA resistance.

The vagus, superior laryngeal and glossopharyngeal nerves were carefully exposed bilaterally, marked with short lengths of cotton thread and maintained intact for later sectioning. The geniohyoid muscle, a UA dilator muscle, was exposed and fine bipolar copper wire electrodes were inserted to record electrical activity. The preparation is shown schematically in figure 1 . The geniohyoid muscle electromyogram (EMG) wires were connected to an amplifier and integrator system (Neurolog system NL 900A, Digitimer, Welwyn Garden, UK). The signal was band pass filtered, rectified and amplified (Neurolog NL 104) before being integrated (Neurolog NL 703). All signals were digitized and recorded using a commercial data-acquisition system and stored for later analysis on a microcomputer.

Table 1. - Effect of cool air on ventilation and upper airway resistance

\begin{tabular}{lccccccc}
\hline & $\begin{array}{c}f \mathrm{R} \\
\text { breaths } \cdot \mathrm{min}^{-1}\end{array}$ & $\begin{array}{c}V \mathrm{~T} \\
\mathrm{~mL} \cdot 100 \mathrm{~g}-1\end{array}$ & $\begin{array}{c}V^{\prime} \mathrm{E} \\
\mathrm{mL} \cdot \mathrm{min}^{-1} \cdot 100 \mathrm{~g}-1\end{array}$ & $\begin{array}{c}t \mathrm{I} \\
\mathrm{s}\end{array}$ & $\begin{array}{c}t \mathrm{E} \\
\mathrm{s}\end{array}$ & $\begin{array}{c}\text { UARN } \\
\mathrm{cmH}_{2} \mathrm{O} \cdot \mathrm{L}^{-1} \cdot \mathrm{s}^{-1} \cdot \mathrm{kg}^{-1}\end{array}$ & $\begin{array}{c}\text { UATEMP } \\
{ }^{\circ} \mathrm{C}\end{array}$ \\
\hline Control & $42.8 \pm 14.0$ & $1.03 \pm 0.32$ & $45.6 \pm 13.7$ & $0.53 \pm 0.25$ & $1.11 \pm 0.56$ & $64.3 \pm 14.6$ & $36.1 \pm 1.4$ \\
Cool air & $32.8 \pm 11.1^{*}$ & $1.06 \pm 0.29$ & $38.7 \pm 13.8 *$ & $0.55 \pm 0.23$ & $1.36 \pm 0.57^{*}$ & $52.5 \pm 8.3^{*}$ & $26.7 \pm 1.8^{*}$ \\
\hline
\end{tabular}

Values are presented as mean \pm SD. $f \mathrm{R}$ : respiratory frequency; $V$ T: tidal volume; $V$ 'E: minute ventilation; $t \mathrm{I}$ : inspiratory duration; $t \mathrm{E}$ : expiratory duration; UARN: upper airway resistance: UATEMP: upper airway temperature. Measurements were taken during application of warm air (control) and cool air to the isolated upper airway in 17 animals with the nose open and all nerves intact. *: p<0.05, compared with control (by analysis of variance).

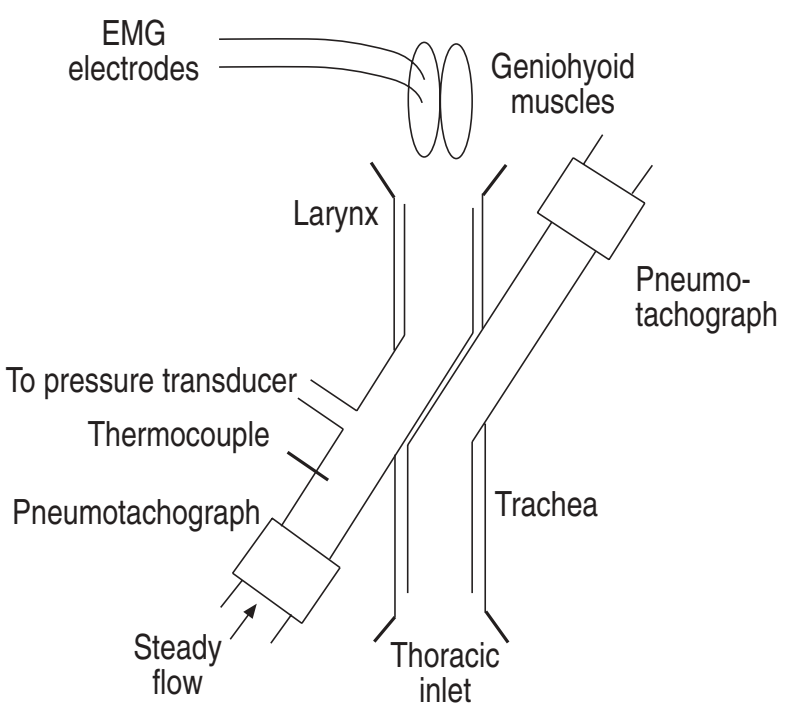
geniohyoid electromyographic (EMG) activity, ventilation and isolated upper airway resistance.

\section{Protocol}

In all 17 animals, with the nose open and the vagus, superior laryngeal and glossopharyngeal nerves intact, variables were recorded continuously during the application of warmed, humidified air. Trials were performed by switching to cool, room-humidity air, which was applied for $40 \mathrm{~s}$ before switching back to warm air. The vagus nerves were cut at the mid-cervical level and the coolair trial was repeated in all 17 animals. Following this, in eight of the 17 animals, the nose was sealed, the superior laryngeal and glossopharyngeal nerves were cut and the cool-air trial was repeated. In the remaining nine animals, a solution of $2 \%$ xylocaine was applied to the entire UA and the cool-air trial repeated after $10 \mathrm{~min}$.

\section{Data analysis}

Values for respiratory variables, UA resistance and geniohyoid muscle activity were averaged from 10 control breaths before the application of cool air to the UA, from 10 breaths during the trial period when the effect of cooling was greatest and from 10 breaths following removal of the test gas. Data are expressed in absolute values or as per cent change with respect to control \pm SD. Data were analysed for statistical significance using analysis of variance (ANOVA) and Fisher's least significant difference test, with p-values $<0.05$ being taken as significant.
Fig. 1. - Diagram of the experimental technique used to measure 


\section{Results}

Cooling of the UA had no effect on body temperature, UA airflow or arterial blood pressure. With the nose open and with the vagus, superior laryngeal and glossopharyngeal nerves intact, there was no phasic EMG activity in the geniohyoid muscle. Values for respiratory variables and for UA resistance before and during the application of cool air to the UA are shown in table 1, and responses to cooling are shown as per cent changes from baseline warm-air values for UA resistance in figure $2 \mathrm{a}$ and for respiratory variables in figure $3 \mathrm{a}$. These data show that cooling produced a significant fall in UA resistance. Cooling also significantly decreased minute ventilation. This was due to a significant decrease in respiratory frequency whereas tidal volume was unaffected. The effect on respiratory frequency was due to a significant prolongation of expiratory time without any change in inspiratory time. A typical example of the effect of cooling on ventilation and UA resistance is shown in figure 4. At the onset of UA cooling, there was a rapid decrease in respiratory frequency but no change in tidal volume. Initially, there was a slight, short-lived increase in UA pressure, which was followed by a sustained fall in pressure.
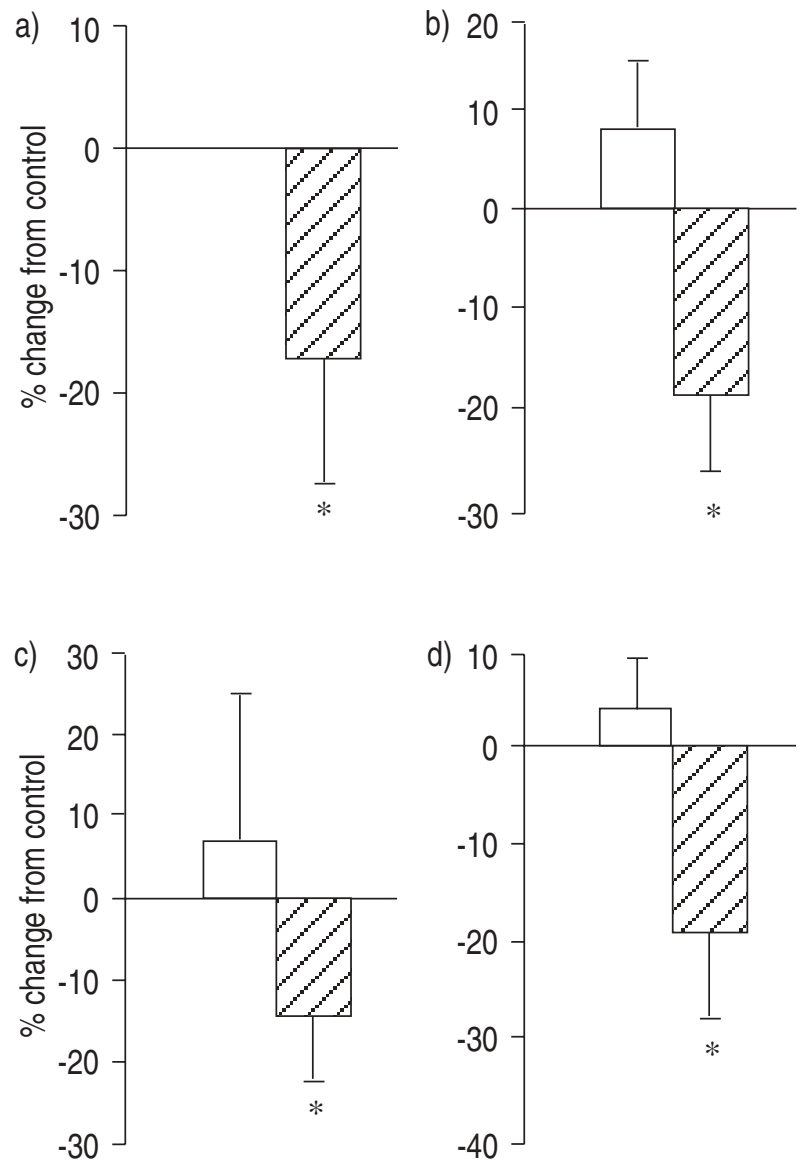

Fig. 2. - Effects of upper airway cooling on geniohyoid electromyographic activity $(\square)$ and upper airway resistance $(\square)$. a) Nose open and vagus, superior laryngeal and glossopharyngeal nerves intact; $b$ ) nose open and vagi cut; c) nose closed and vagus, superior laryngeal and glossopharyngeal nerves cut; and d) nose open and topical anaesthesia of the upper airway. Values are expressed as percentage change from warm air control \pm SD. $*: \mathrm{p}<0.05$, compared with control (by analysis of variance).
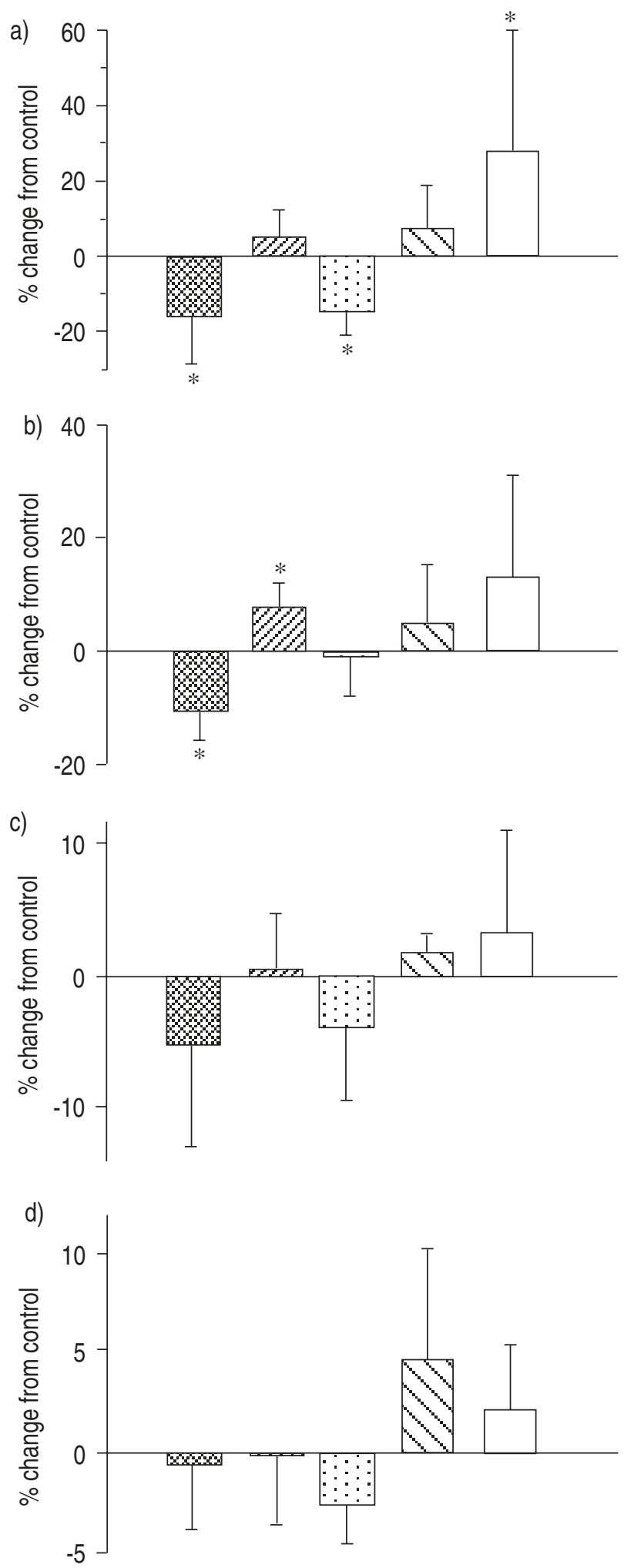

Fig. 3. - Effects of upper airway cooling on respiratory frequency ( $)$ ), tidal volume $(\square)$, ventilation $(\because)$, inspiratory duration $(\square)$ and expiratory duration $(\square)$. a) Nose open and vagus, superior laryngeal and glossopharyngeal nerves intact; b) nose open and vagi cut; c) nose closed and vagus, superior laryngeal and glossopharyngeal nerves cut; and d) nose open and topical anaesthesia of the upper airway. Values are expressed as percentage change from warm air control \pm SD. $*: \mathrm{p}<0.05$, compared to control (by analysis of variance). 
a)

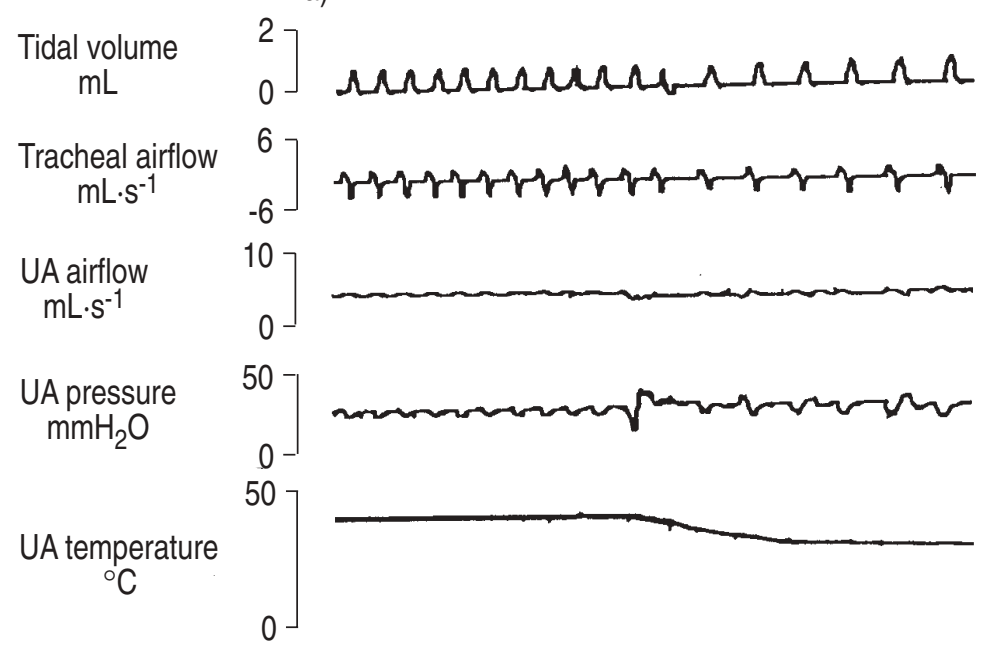

b)

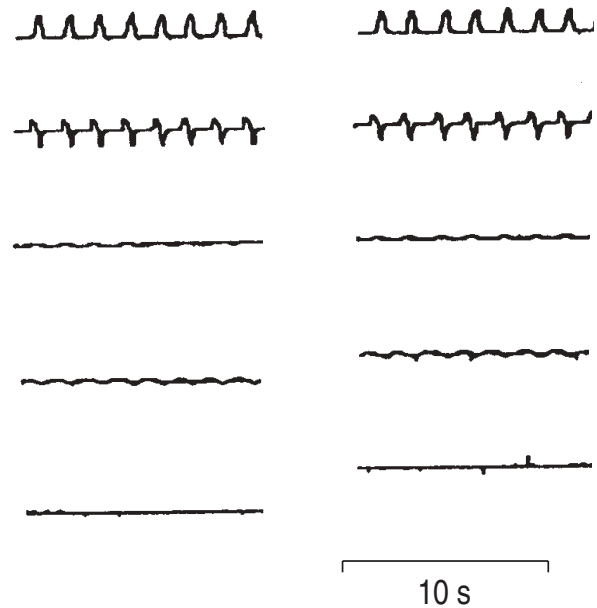

Fig. 4. - Simultaneous recordings of ventilation and upper airway (UA) airflow, pressure and temperature: a) before and during the onset of UA cooling; b) during UA cooling; and c) after UA cooling with the nose open and the vagus, superior laryngeal and glossopharyngeal nerves intact.

Bilateral section of the vagus nerves had no significant effect on UA resistance but caused a significant fall in respiratory frequency $(55.6 \pm 21.5 \%$ of intact $)$ and a significant increase in tidal volume $(215.3 \pm 62.1 \%$ of intact). Phasic inspiratory geniohyoid EMG activity became apparent in 12 out of 17 animals. Following vagotomy, cooling caused a significant decrease in UA resistance and a significant increase in geniohyoid EMG activity (fig. 3b). Cooling also caused a significant decrease in respiratory frequency and a significant increase in tidal volume, whereas minute ventilation, inspiratory time and expiratory time were un-affected (fig. 3b).

In eight out of the 17 vagotomized animals, the superior laryngeal and glossopharyngeal nerves were then cut bilaterally and the nose was sealed. This caused a significant increase in baseline UA resistance $(246.3 \pm 82.1 \%$ of nose open, vagi cut values, $n=8$ ) but had no effect on baseline ventilatory variables or geniohyoid EMG activity. Following these interventions, cooling still caused a significant decrease in UA resistance (fig. 2c) but the effects on geniohyoid EMG activity (fig. 2c) and on ventilation (fig. 3c) were abolished.

In nine out of the 17 vagotomized animals, topical anaesthesia of the entire UA had no effect on baseline UA resistance, geniohyoid EMG activity or ventilatory variables. Cooling still significantly reduced UA resistance (fig. 2d) but the effects of cooling on geniohyoid EMG activity and on ventilatory variables observed before airway anaesthesia were abolished (figs. $2 \mathrm{~d}$ and $3 \mathrm{~d}$ ).

\section{Discussion}

The main findings of this study are that moderate cooling of the UA reduces UA resistance and excites geniohyoid muscle activity in young guinea-pigs. With the vagi intact, no phasic geniohyoid muscle activity could be detected, but phasic inspiratory activity became apparent following vagotomy. This is consistent with the observation that UA muscle activity is enhanced by vagotomy in adult animals [22, 23] and suggests that the Hering-Breuer inflation reflex may have a greater inhibitory effect on UA muscle activity in young animals since geniohyoid activity was absent with intact vagi in the present experiments. In this regard, it is known that the inspiratory-inhibiting component of the Hering-Breuer reflex is more active in newborns than in adults [24]. This reflex may also explain why cooling in the present experiments had no effect on tidal volume in vagi-intact animals but caused an increase in tidal volume following vagotomy.

In the present experiments, UA resistance was unaffected by vagotomy, despite the increase in geniohyoid activity. This may be because any tendency for the resistance to decrease as a result of geniohyoid contraction was counteracted by the tendency for resistance to increase owing to interruption of vagal efferents coursing through the recurrent laryngeal nerves to the laryngeal muscles [8]. However, the effects of such efferents on UA resistance in young guinea-pigs are unknown. Cutting the recurrent laryngeal nerves causes approximately a 30\% increase in laryngeal resistance in anaesthetized adult rats [8] but only a slight increase in laryngeal resistance in anaesthetized adult dogs [25].

The excitatory effect of UA cooling on geniohyoid muscle activity is consistent with observations in adult rats, where moderate cooling also increased geniohyoid muscle activity [9]. However, UA cooling has been shown to inhibit laryngeal muscle activity in adult cats [10], to have no effect on posterior cricoarytenoid muscle activity but to inhibit its response to UA occlusion in adult dogs [11], to excite genioglossus muscle activity in humans [26] and to either excite or inhibit hypoglossal nerve (the main motor nerve supply to the UA muscles) activity in adult cats [7]. These inconsistent findings may be due to species differences, differences in anaesthesia and different methodologies, particularly the wide range of temperatures used. Anaesthesia is known preferentially to depress UA muscle activity [27]. However, the excitatory effect of cool air on UA muscle activity observed in the present experiments in anaesthetized young guinea-pigs and in our previous study in anaesthetized adult rats [8] is consistent with the excitation of UA muscle activity caused by UA cooling in 
unanaesthetized humans [26] and decerebrate cats [7]. The continuous expiratory airflow used in the present experiments differs from the oscillatory flow normally occurring in the UA. Mortola and Rezzonico [28] compared the effects of continuous and oscillatory flow on ventilation in kittens and found that the ventilatory inhibition observed with continuous flow was not obtained with oscillatory flow. However, these authors did not measure UA temperature, and therefore the reason for the absence of a ventila-tory response to oscillatory flow is unclear.

Topical anaesthesia of the UA or sealing the nose and cutting the superior laryngeal and glossopharyngeal nerves abolished the increase in geniohyoid activity caused by cooling. These procedures were carried out to minimize afferent activity from the larynx, pharynx and nose, and suggest that the excitatory effect is a reflex from UA receptors. These procedures also abolished the ventilatory effects of UA cooling, and therefore the geniohyoid activation may be secondary to the ventilatory effects observed before sealing the nose and with the nerves intact.

The effects of UA cooling on UA resistance have not been examined previously in young animals. At the onset of UA cooling, there was usually a slight, short-lived increase in UA pressure. The cause of this is unclear but it may have been secondary to the inhibition of breathing since it was not observed when ventilatory effects were abolished. In this regard, it is worth noting that MoRToLA and REzzonico [28] found that high oscillatory flows caused closure of the UA in kittens. Following this initial slight increase in UA resistance, cooling caused a larger sustained decrease in UA resistance, which was not abolished by topical UA anaesthesia or the combination of nose sealing and section of the superior laryngeal and glossopharyngeal nerve. In adult cats, laryngeal cooling has been shown to reduce laryngeal resistance, and this effect was greater following superior laryngeal nerve section [10]. We have previously reported that UA cooling re-duces UA resistance in adult rats and that this effect is largely not of reflex origin [8]. Therefore, although cooling increases UA dilator muscle activity, the fall in resistance appears to be due mainly to some other effect. It is proposed that cooling causes constriction of UA mucosal blood vessels, leading to a thinning of the mucosa, an increase in luminal cross-sectional area and a decrease in resistance. This is supported by the recent report that cooling decreases tracheal mucosal blood flow in humans [29]. The effect of changes in UA mucosal blood flow on UA cross-sectional area and resistance have been well described previously [30-33]. Another possible mechanism could be drying and shrinking of the mucosa due to the lower humidity of the cool air.

It is possible that the greater temperature and humidity of the warm compared with the cool air may have slightly affected airflow in the UA so that the actual UA airflow may have been slightly greater than the measured flow during warm airflow. From Bernoulli's principle, this would have resulted in a reduction in UA pressure so that switching to cool air would have caused an increase in UA pressure. However, a decrease in UA pressure was observed, and therefore the fall in UA resistance is unlikely to be an artefact of switching from warm to cool air.

In summary, this investigation has demonstrated that moderate upper airway cooling increases geniohyoid muscle activity and reduces upper airway resistance in young guinea-pigs. The geniohyoid excitation is abolished by the elimination of upper airway receptor activity but the fall in upper airway resistance is not abolished, and may be due to a direct effect of cooling on upper airway mucosal blood flow.

Acknowledgements: The authors wish to thank $\mathrm{T}$. Dowling and J. Slattery for technical assistance.

\section{References}

1. Sant'Ambrogio G, Mathew OP, Sant'Ambrogio FB, Fisher JT. Laryngeal cold receptors. Respir Physiol 1985; 59: 35-44.

2. Sant'Ambrogio G, Brambilla-Sant'Ambrogio F, Mathew OP. Effect of cold air on laryngeal mechanoreceptors in the dog. Respir Physiol 1986; 64: 45-56.

3. Mortola JP, Citterio G, Agostoni E. Sulphur dioxide block of laryngeal receptors in rabbits. Respir Physiol 1985; 62: 195-202.

4. Tsubone H. Nasal "flow" receptors of the rat. Respir Physiol 1989; 75: 51-64.

5. Bradford A, Nolan P, O'Regan RG, McKeogh D. Carbon dioxide-sensitive superior laryngeal nerve afferents in the anaesthetised cat. Exp Physiol 1993; 78: 787-798.

6. Orani GP, Anderson JW, Sant'Ambrogio G, Sant'Ambrogio FB. Upper airway cooling and L-menthol reduce venti-lation in the guinea pig. J Appl Physiol 1991; 70: 20802086.

7. Ukabam CV, Knuth SL, Bartlett D Jr. Phrenic and hypoglossal neural responses to cold airflow in the upper airway. Respir Physiol 1992; 87: 157-164.

8. O'Halloran KD, Curran AK, Bradford A. Ventilatory and upper-airway resistance responses to upper-airway cooling and $\mathrm{CO}_{2}$ in anaesthetised rats. Eur J Physiol 1994; 429: 262-266.

9. O'Halloran KD, Curran AK, Bradford A. Effect of upper airway cooling and $\mathrm{CO}_{2}$ on diaphragm and geniohyoid muscle activity in the rat. Eur Respir J 1996; 9: 2323 2327.

10. Jammes Y, Barthelemy P, Delpierre S. Respiratory effects of cold air breathing in anaesthetised cats. Respir Physiol 1983; 54: 41-54.

11. Mathew OP, Sant'Ambrogio FB, Sant'Ambrogio G. Effects of cooling on laryngeal reflexes in the dog. Respir Physiol 1986; 66: 61-70.

12. Fisher JT, Mathew OP, Sant'Ambrogio FB, Sant'Ambrogio G. Reflex effects and receptor responses to upper airway pressure and flow stimuli in developing puppies. $J$ Appl Physiol 1985; 58: 258-264.

13. Boggs DF, Bartlett D Jr. Chemical specificity of a laryngeal apneic reflex in puppies. J Appl Physiol 1982; 53: 455-462.

14. Harding R, Johnson P, McClelland ME. Liquid-sensitive laryngeal receptors in the developing sheep, cat and monkey. J Physiol (Lond) 1978; 277: 409-422.

15. Mathew OP, Anderson JW, Orani GP, Sant'Ambrogio FB, Sant'Ambrogio G. Cooling mediates the ventilatory depression associated with airflow through the larynx. Respir Physiol 1990; 82: 359-368.

16. Curran AK, O'Halloran KD, Bradford A. Effects of superior laryngeal nerve section on ventilation in neonatal guinea-pigs. Respir Physiol 1995; 101: 23-29.

17. Al-Shway SF, Mortola JP. Respiratory effects of airflow through the upper airways in newborn kittens and puppies. J Appl Physiol 1982; 53: 805-814. 
18. Sant'Ambrogio FB, Anderson JW, Sant'Ambrogio G. Menthol in the upper airway depresses ventilation in newborn dogs. Respir Physiol 1992; 89: 299-307.

19. Dawes DS. Foetal and Neonatal Physiology. Chicago, IL, Year Book Medical Publishers, 1968; pp. 225-227.

20. Curran AK, O'Halloran KD, Bradford A. Developmental changes in the effect of airway $\mathrm{CO}_{2}$ on ventilation in anaesthetized guinea-pigs. J Physiol (Lond) 1995; 489P: $163 \mathrm{P}$.

21. Zhang H-Q, Tai H-H, Lai Y-L. Age-dependent mechanism in guinea pig bronchoconstriction induced by exsanguination. Respir Physiol 1995; 99: 361-369.

22. Brouillette RT, Thach BT. Control of genioglossus muscle inspiratory activity. J Appl Physiol 1980; 49: 801-808.

23. O'Halloran KD, Curran AK, Bradford A. Effect of almitrine on ventilation and on diaphragm and geniohyoid muscle activity in the rat. Clin Sci 1996; 91: 337-345.

24. Bryan AC, Bowes G, Maloney JE. Control of breathing in the fetus and the newborn. In: Cherniak NS, Widdicombe JG, eds. Handbook of Physiology, Section 3: The Respiratory System, Volume II: Control of Breathing, Part 2. Washington, DC, American Physiological Society, 1986; 621-646.

25. McCaffrey TV, Kern EB. Laryngeal regulation of airway resistance I. Chemoreceptor reflexes. Ann Otol Rhinol Laryngol 1980; 89: 462-466.

26. Basner RC, Ringler J, Berkowitz S, et al. Effect of in- spired air temperature on genioglossus activity during nose breathing in awake humans. J Appl Physiol 1990; 69: $1098-1103$.

27. Hwang J-C, St John WM, Bartlett D Jr. Respiratoryrelated hypoglossal nerve activity: influence of anesthetics. J Appl Physiol 1983; 55: 785-792.

28. Mortola JP, Rezzonico R. Ventilation in kittens with chronic section of the superior laryngeal nerves. Respir Physiol 1989; 76: 369-382.

29. Le Merre C, Kim HH, Chediak AD, Wanner A. Airway blood flow responses to temperature and humidity of inhaled air. Respir Physiol 1996; 105: 235-239.

30. Lung MA, Wang JCC. Autonomic nervous control of nasal vasculature and airflow resistance in the anaesthetized dog. J Physiol (Lond) 1989; 419: 121-139.

31. Parisi RA, Wasicko MJ, Hutt DA, Mandel M, Santiago TV, Edelman, NH. Mucosal vasoconstriction increases pharyngeal size in normal subjects. Am Rev Respir Dis 1989; 139: A374.

32. Wasicko M, Hutt DA, Parisi RA, Neubauer JA, Mezrich $\mathrm{R}$, Edelman NH. The role of vascular tone in the control of upper airway collapsibility. Am Rev Respir Dis 1990; 141: 1569-1577.

33. Wasicko MJ, Leiter JC, Erlichman JS, Strobel RJ, Bartlett D Jr. Nasal and pharyngeal resistance after topical mucosal vasoconstriction in normal humans. Am Rev Respir Dis 1991; 144: 1048-1052. 